\title{
Radiological differences in computed tomography findings and texture analysis between cystic lymph node metastases of human papillomavirus-positive oropharyngeal cancer and second branchial cysts
}

\author{
Akira Baba ${ }^{1 A, B, C, D, E, F}$, Hisashi Kessoku ${ }^{2 B}$, Ryo Kurokawa ${ }^{3 C, E}$, Hideomi Yamauchii ${ }^{1 D}$, Taisuke Akutsu ${ }^{2 B}$, Eiji Shimura ${ }^{2 B}$, \\ Koshi lkeda ${ }^{1 \mathrm{E}}$, Hiroya Ojiri ${ }^{1 \mathrm{E}}$ \\ 'Department of Radiology, The Jikei University School of Medicine, Japan \\ ${ }^{2}$ Department of Otorhinolaryngology, The Jikei University School of Medicine, Japan \\ ${ }^{3}$ Department of Radiology, Graduate School of Medicine, The University of Tokyo, Japan
}

\section{Abstract}

Purpose: The study aimed to analyse radiological differences in computed tomography (CT) findings and texture analysis between cystic lymph node metastases (CNM) in human papillomavirus (HPV)-positive oropharyngeal cancer $(\mathrm{OPC})$ and second branchial cleft cysts $\left(2^{\text {nd }} \mathrm{BC}\right)$.

\begin{abstract}
Material and methods: Patients with pathological evidence of CNM-HPV-OPC and $2^{\text {nd }} \mathrm{BC}$, who underwent contrastenhanced CT, were retrospectively evaluated. The evaluated characteristics include age, sex, and CT findings. CT findings included the maximum and minimum transverse diameters, maximum caudal diameter, thickness of the peripheral wall, presence of internal septation, presence of surrounding fat stranding, location, and 40 texture parameters.
\end{abstract}

Results: A total of 13 patients had CNM-HPV-OPC (19 lesions), while 20 patients had $2^{\text {nd }} \mathrm{BC}$ (20 lesions). Patients with $2^{\text {nd }} \mathrm{BC}$ were significantly younger than those with CNM-HPV-OPC $(p<0.001)$. In terms of diameter, $2^{\text {nd }} \mathrm{BC}$ lesions were significantly larger than the CNM-HPV-OPC lesions $(p<0.001)$. CNM-HPV OPC lesions had significantly thicker walls than $2^{\text {nd }} \mathrm{BC}$ lesions $(p<0.001)$. CNM-HPV-OPC lesions had significantly higher association with internal septations than $2^{\text {nd }} \mathrm{BC}$ lesions $(p<0.001)$. Second BC lesions were significantly less common at level III than CNM-HPV-OPC lesions ( $p=0.047)$. Among the 40 texture parameters measured, 8 had significant differences $(p \leq 0.001)$.

Conclusions: There were significant differences in CT findings and textural parameters between CNM-HPV-OPC and $2^{\text {nd }} \mathrm{BC}$ lesions. These results may help in differentiating one from the other.

Key words: human papillomavirus-positive oropharyngeal cancer, cystic lymph node metastases, computed tomography, texture analysis.

\section{Introduction}

In the $8^{\text {th }}$ edition of the American Joint Committee on Cancer (AJCC) staging manual, oropharyngeal cancers were divided into human papillomavirus (HPV)-positive and HPV-negative carcinomas [1]. Cervical lymph node metastases in HPV-positive oropharyngeal carcinomas (OPC) are often identified as cystic lymph node masses. Cystic lesions of the neck may be detected on imaging in patients who present with palpable neck masses and unremarkable medical history. In these cases, radiologists experienced difficulties in differentiating cystic lymph

Correspondence address:

Akira Baba, Department of Radiology, The Jikei University School of Medicine, Japan, e-mail: akirababa@jikei.ac.jp

Authors' contribution:

A Study design · B Data collection · C Statistical analysis · D Data interpretation · E Manuscript preparation · F Literature search · G Funds collection 
node metastases in HPV-positive oropharyngeal cancer (CNM-HPV-OPC) from other cystic neck lesions. In particular, the second branchial cleft cyst $\left(2^{\text {nd }} \mathrm{BC}\right)$ resembled CNM-HPV-OPC in clinical presentation and radiological findings. A previous study compared computed tomography (CT) imaging findings between cystic cervical lymph node metastases and $2^{\text {nd }} \mathrm{BC}$ [2]. However, this study included nasopharyngeal and oropharyngeal cancers, whose HPV status was unknown. Previous studies have not compared the radiological differences and texture analysis between CNM-HPV-OPC and $2^{\text {nd }}$ BCs in a population of HPV-positive patients. Therefore, we aimed to investigate radiological differences in $\mathrm{CT}$ findings and texture analysis between CNM-HPV-OPC and $2^{\text {nd }} \mathrm{BCs}$.

\section{Material and methods}

Data of patients with all pathologically proven CNM$\mathrm{HPV}-\mathrm{OPC}$ and $2^{\text {nd }} \mathrm{BC}$ in the postoperative period, who underwent contrast-enhanced CT scanning for pretreatment evaluation between May 2009 and June 2019 in our institution, were retrospectively evaluated. Patients with lesions of size less than $1.5 \mathrm{~cm}$ in length and strong artifacts were excluded. Cystic lesions were defined as those that included more than $30 \%$ of the low-density area. Because previous studies used a cut-off value of $20 \%$ [3], we used a cut-off value of $30 \%$ in the present study to make the definition as cyst lesions more consistent. The design of this study was approved by our Institutional Review Board and Ethics Committee (approval number: 31-083 [9582]). The requirement for informed consent was waived because this was a retrospective study.

The CT examinations were performed using a 128channel multidetector CT scanner (SOMATOM Definition Flash; Siemens) with the following standard settings: $128 \times 0.6 \mathrm{~mm}$ collimation with $0.5 \mathrm{~mm}$-thick sections, $3 \mathrm{~mm}$ reconstruction, and a pitch of 1.0. All CT scans in this study were performed in the supine position.

Two radiologists specializing in the head and neck region retrospectively evaluated the imaging findings. The evaluated characteristics were age, sex, and CT findings, including maximum transverse diameter, minimum transverse diameter, maximum cranio-caudal diameter, thickness of the peripheral wall, presence of internal septation, presence of surrounding fat stranding, and location.

Texture parameters from texture analysis were calculated using LIFEx software (https://lifexsoft.org/).

The $\mathrm{DICOM}^{\circledR}$ data was adapted. Before feature extraction, all images underwent standardized preprocessing as follows: spatial resampling to $0.4 \times 0.4 \times 3 \mathrm{~mm}$, intensity discretization to 128 grey levels, and relative intensity rescaling by a mean of \pm 3 standard deviations. Two-dimensional region of interest (ROI) along the margin of the lesion was measured in each axial image, the $3 \mathrm{D}$ volume of interest (VOI) was generated from the data, and 40 texture parameters (HISTO_Skewness, HISTO_
Kurtosis, HISTO_Entropy_log10, HISTO_Entropy_log2, HISTO_Energy, SHAPE_Volume, SHAPE_Sphericity, SHAPE_Compacity, GLCM_Homogeneity, GLCM_ Energy, GLCM_Contrast, GLCM_Correlation, GLCM_ Entropy_log10, GLCM_Entropy_log2, GLCM_Dissimilarity, GLRLM_SRE, GLRLM_LRE, GLRLM_LGRE, GLRLM_HGRE, GLRLM_SRLGE, GLRLM_SRHGE, GLRLM_LRLGE, GLRLM_LRHGE, GLRLM_GLNU, GLRLM_RLNU, GLRLM_RP, NGLDM_Coarseness, NGLDM_Contrast, NGLDM_Busyness, GLZLM_ SZ, GLZLM_LZE, GLZLM_LGZE, GLZLM_HGZE, GLZLM_SZLGE, GLZLM_SZHGE, GLZLM_LZLGE, GLZLM_LZHGE, GLZLM_GLNU, GLZLM_ZLNU, and GLZLM_ZP) were calculated for each 3D-VOI. For patients with multiple lesions, the $3 \mathrm{D}$-VOI was set for each lesion, and the texture parameters of each lesion were calculated.

\section{Statistical analysis}

Student's $t$-test was performed to compare age, while Mann-Whitney's $U$ test was performed to compare the maximum and minimum transverse diameters, maximum caudal diameter, thickness of the peripheral wall, and texture parameters. Lastly, Fisher's exact test was used to compare sex, presence of internal septations, presence of surrounding fat stranding, and lesion location. The results were considered significant after Bonferroni correction ( $p<0.05 / 40=0.00125$ for texture parameters). In the other results, $p$-values of less than 0.05 were considered to indicate statistical significance. All statistical analyses were performed using BellCurve for Excel (SSRI, Tokyo, Japan).

\section{Result}

Thirteen patients had CNM-HPV-OPC (19 lesions), while 20 had $2^{\text {nd }}$ BC (20 lesions). The results for age, sex, and CT findings are shown in Table 1. Patients with CNM-HPVOPC were significantly older than those with $2^{\text {nd }} B C$ $(p<0.001)$. Maximum transverse diameter, minimum transverse diameter, and maximum caudal diameter were significantly smaller in those with CNM-HPV-OPC lesions than in those with $2^{\text {nd }} \mathrm{BC}$ lesions $(p<0.001)$. The thickness of the peripheral wall was significantly higher in those with CNM-HPV-OPC lesions than in those with $2^{\text {nd }} \mathrm{BC}$ lesions $(p<0.001)$. Regarding the presence of internal septations, CNM-HPV-OPC lesions tended to have a significantly higher association with internal septations than did $2^{\text {nd }} \mathrm{BC}$ lesions $(p<0.001)$. With regard to the location, CNM-HPV-OPC lesions were significantly more common at level III than were the $2^{\text {nd }}$ BC lesions $(p=0.047)$. There were no significant differences in sex or presence or absence of ambient fatty acid opacity $(p=0.16-0.20)$. Of the 40 texture parameters measured, significant differences were found in $8(p \leq 0.001)$ (Table 2$)$. 


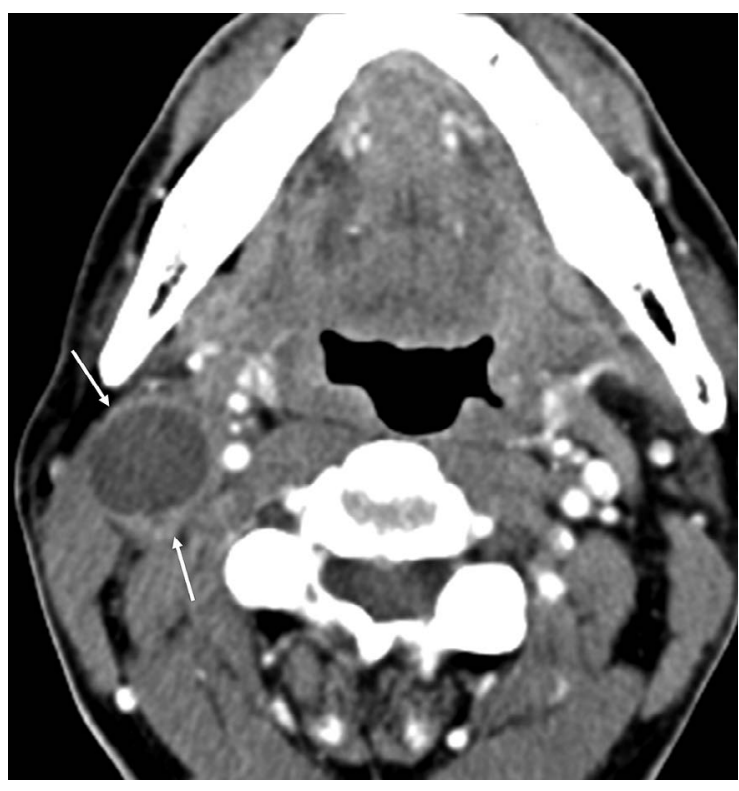

Figure 1. Cystic lymph node metastasis in human papillomavirus- positive oropharyngeal cancer. Contrast-enhanced computed tomography of a male patient in his 50 s showing cystic lymphadenopathy with a slightly thick peripheral wall at level II on the right side

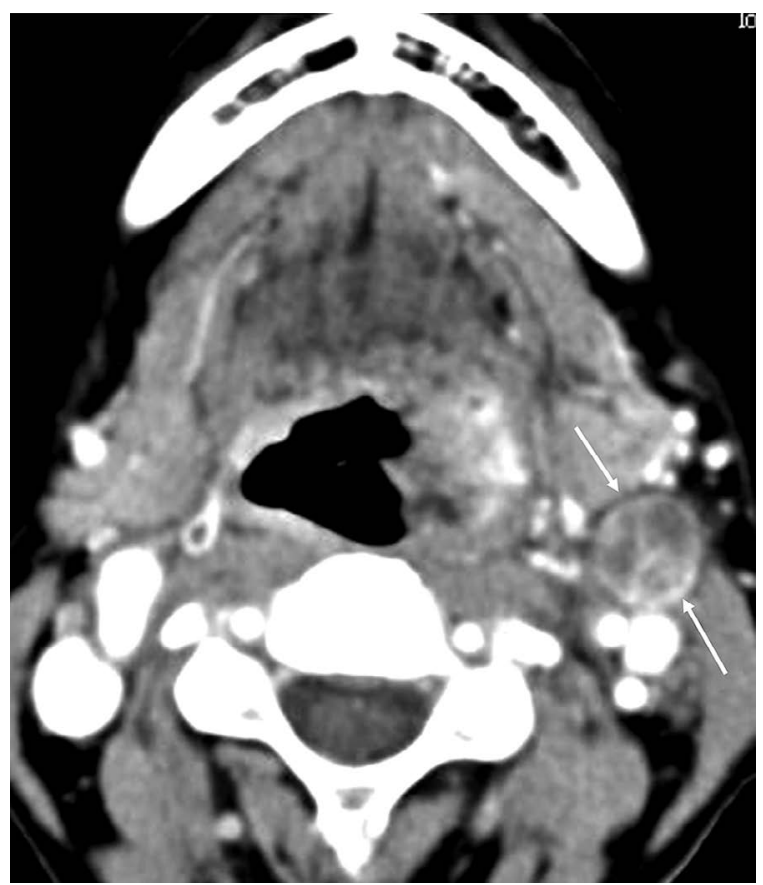

Figure 3. Cystic lymph node metastasis in human papillomavirus-positive oropharyngeal cancer. Contrast-enhanced computed tomography of a female patient in her 70s showing cystic lymphadenopathy (arrow) with internal septations at level II on the left side

\section{Discussion}

In the present study, significant differences in age, size, thickness of the peripheral wall, presence of internal septations, location, and multiple textural parameters were observed between CNM-HPV-OPC and $2^{\text {nd }} \mathrm{BC}$. Although differentiating between CNM-HPV-OPC and $2^{\text {nd }} \mathrm{BC}$ on

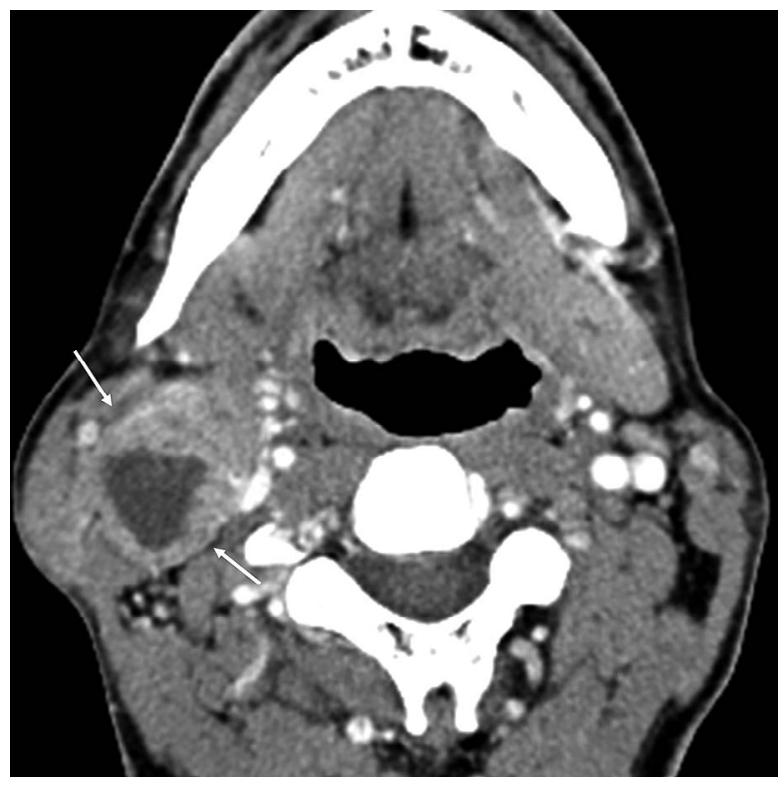

Figure 2. Cystic lymph node metastasis in human papillomavirus-positive oropharyngeal cancer. Contrast-enhanced computed tomography of a male patient in his 60s showing cystic lymphadenopathy (arrow) with a thick and irregular peripheral wall at level II on the right side

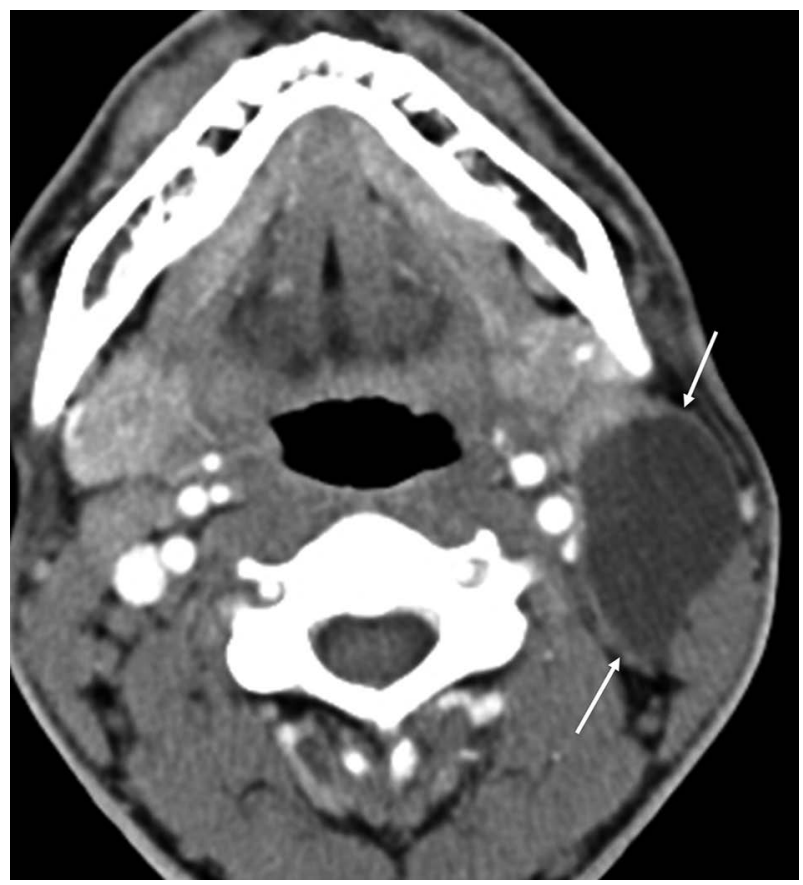

Figure 4. Second branchial cleft cyst. Contrast-enhanced computed tomography of a female patient in her 20s showing a cystic lesion (arrow) with a well-defined and smooth margin at level II on the left side and posterior to the left submandibular gland, lateral to the carotid sheath, and medial to the sternocleidomastoid muscle

the basis of CT imaging is possible to some extent, differences in texture parameters may further improve diagnostic accuracy.

HPV-positive oropharyngeal cancer is a newly defined category of oropharyngeal cancer in the AJCC cancer staging manual, $8^{\text {th }}$ edition [1]. Compared with HPVnegative oropharyngeal cancer, the HPV-positive one is 


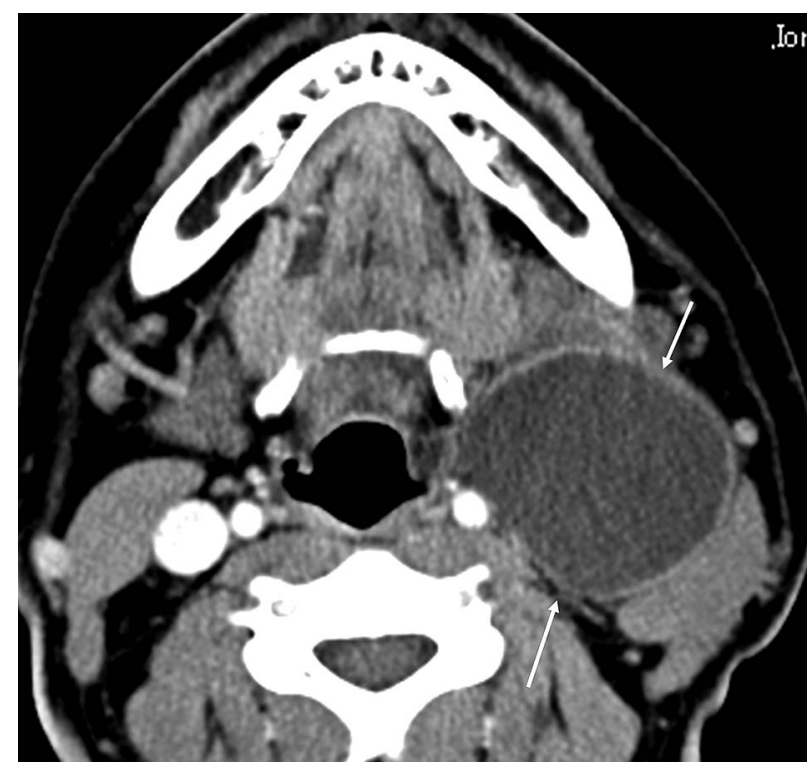

Figure 5. Second branchial cleft cyst. Contrast-enhanced computed tomography of a female patient in her 20s showing a cystic lesion (arrow) with a well-defined and smooth margin and a slightly thick peripheral wall at level II, on the left side and posterior to the left submandibular gland, lateral to the carotid sheath, and medial to the sternocleidomastoid muscle

more responsive to treatment and has a better prognosis. Moreover, the number of cases has increased in recent years $[4,5]$. It also tends to be more common in relatively young people and was reported to be related to sexual activity $[4,5]$. CNM-HPV-OPC may sometimes present as a palpable, painless lateral neck mass. The imaging findings of CNM-HPV-OPC are characterized by a higher frequency of cystic cervical lymph node metastases than those of HPV-negative oropharyngeal carcinoma [6-8]. The $2^{\text {nd }} \mathrm{BC}$ is a congenital benign cervical cystic lesion arising from congenital remnants of the second branchial cleft, and it accounts for the vast majority of branchial cleft anomalies. The Bailey type II lesion (lesion located anterior to the sternocleidomastoid muscle, posterior to the submandibular gland, and lateral to the carotid sheath) is the most common one in this entity. Clinically, the $2^{\text {nd }} \mathrm{BC}$ presents as a painless lesion without superinfection in the lateral neck, such as CNM-HPV-OPC. The imaging findings of $2^{\text {nd }} \mathrm{BCs}$ include unilocular cystic lesions with relatively well-defined and smooth margins. As described above, the imaging findings of CNM-HPVOPC and $2^{\text {nd }} \mathrm{BCs}$ are similar to cervical cystic lesions, and both diseases present as painless cervical cystic lesions. These similarities make the clinical and radiological diagnoses of these diseases difficult. Because CNM-HPV-OPC is a metastatic lesion of a malignant tumour and $2^{\text {nd }} \mathrm{BCs}$ is a benign lesion, it is clinically important to differentiate between the 2 lesions using imaging diagnosis.

A previous study on the differentiation of benign and malignant cystic cervical lesions reported that cystic cervical lymph node metastases tended to be older, more heterogeneous in internal texture, smaller in size, and have more surrounding fat stranding and internal septum than

Table 1. Attribute data and computed tomography findings of patients with cystic lymph node metastasis of human papillomavirus-positive oropharyngeal cancer and $2^{\text {nd }}$ branchial cyst

\begin{tabular}{|c|c|c|c|}
\hline Parameter & CNM-HPV-OPC & $2^{\text {nd }}$ branchial cyst & $p$-value \\
\hline Mean age \pm standard deviation & $58.2 \pm 8.3$ & $37.9 \pm 13.6$ & $<0.001$ \\
\hline \multicolumn{4}{|l|}{ Sex } \\
\hline Female & 9 & 8 & \multirow[t]{2}{*}{0.16} \\
\hline Male & 4 & 12 & \\
\hline \multicolumn{4}{|l|}{ CT findings } \\
\hline Maximum transverse diameter (cm, median [IQR]) & $2.0(1.6-2.6)]$ & $3.9(3.4-4.6)$ & $<0.001$ \\
\hline Minimum transverse diameter $(\mathrm{cm}$, median $[\mathrm{IQR}])$ & $1.8(1.3-2.1)$ & $3.0(2.8-3.5)$ & $<0.001$ \\
\hline Maximum cranio-caudal diameter (cm, median [IQR]) & $3.0(2.1-3.6)$ & $4.8(4.2-5.2)$ & $<0.001$ \\
\hline Thickness of peripheral wall (mm, median [IQR]) & $5.4(4.0-6.4)]$ & $2.1(1.3-2.8)$ & $<0.001$ \\
\hline \multicolumn{4}{|l|}{ Internal septation } \\
\hline Present & 16 & 1 & \multirow[t]{2}{*}{$<0.001$} \\
\hline Absent & 3 & 19 & \\
\hline \multicolumn{4}{|l|}{ Surrounding fat stranding } \\
\hline Present & 12 & 8 & \multirow[t]{2}{*}{0.20} \\
\hline Absent & 7 & 12 & \\
\hline \multicolumn{4}{|l|}{ Location } \\
\hline Level II & 15 & 20 & \multirow[t]{2}{*}{0.047} \\
\hline Level III & 4 & 0 & \\
\hline
\end{tabular}

CNM-HPV-OPC - cystic lymph node metastasis of HPV-positive oropharyngeal cancer. 
Table 2. Significant texture features of cystic lymph node metastasis of HPV-positive oropharyngeal cancer and 2nd branchial cyst

\begin{tabular}{|c|c|c|c|c|c|c|}
\hline Texture features & $\begin{array}{c}\text { Median of CNM-HPV- } \\
\text { OPC values (IQR) }\end{array}$ & $\begin{array}{l}\text { Median of } 2^{\text {nd }} \text { branchial } \\
\text { cyst values (IQR) }\end{array}$ & $p$-value & Cut-off & $\begin{array}{c}\text { AUC (predicting CNM } \\
\text { HPV + OPC) }\end{array}$ & $\begin{array}{l}\text { AUC (predicting } \\
2^{\text {nd }} \text { branchial cyst) }\end{array}$ \\
\hline \multicolumn{7}{|l|}{ Histogram } \\
\hline Kurtosis & $2.87(2.72-3.35)$ & $4.86(4.86-10.09)$ & $<0.001$ & 3.52 & & 0.90 \\
\hline Entropy $\log 10$ & 1.91 (1.89-1.92) & $1.80(1.79-1.87)$ & $<0.001$ & 1.89 & 0.86 & \\
\hline Entropy $\log 2$ & $6.34(6.30-6.36)$ & $5.98(5.97-6.21)$ & $<0.001$ & 6.28 & 0.86 & \\
\hline Energy & $0.0142(0.0137-0.0147)$ & $0.0166(0.0166-0.0221)$ & $<0.001$ & 0.0162 & & 0.88 \\
\hline \multicolumn{7}{|l|}{ GLRLM } \\
\hline GLNU & $214.7(99.7-319.4)$ & $803.6(803.6-1208.4)$ & $<0.001$ & 323.2 & & 0.87 \\
\hline \multicolumn{7}{|l|}{ NGLDM } \\
\hline Busyness & $0.21(0.08-0.26)$ & $0.59(0.59-1.03)$ & $<0.001$ & 0.32 & & 0.88 \\
\hline \multicolumn{7}{|l|}{ GLZLM } \\
\hline LZE & $4.04(3.24-5.18)$ & $7.08(7.08-22.4)$ & $<0.001$ & 6.75 & & 0.90 \\
\hline LZHGE & 18190 (13932-22299) & 28123 (28123-164027) & $<0.001$ & 22378 & & 0.88 \\
\hline
\end{tabular}

$2^{\text {nd }}$ BC lesions [2]. Similarly, in our study, with the exception of the surrounding fat stranding, CNM-HPV-OPC patients significantly tended to be older, had smaller-sized tumours, and had more internal septations than those with $2^{\text {nd }} \mathrm{BCs}$. These differences in terms of imaging results and age are potentially useful for the differential diagnosis of benign and malignant cystic cervical lesions. In previous reports, CNM-HPV-OPC cases were more common at levels II and III and less common at levels I, IV, and V [7]. In the current study, all lesions of CNM-HPV-OPC were detected at levels II and III. All $2^{\text {nd }}$ BCs were detected at level II. The locations of the lesions on imaging provided useful information in differentiating between them.

Texture analysis is a method for quantifying and characterizing the nature of lesions in images [9] and has recently received increasing attention in the diagnostic imaging field. It is a method in which the shading of pixels in the region of interest is numerically measured, and parameters of "non-uniformity" such as uniformity, directionality, and contrast change are calculated. In recent years, texture analysis has been studied to predict outcomes and survival of patients with head and neck cancer, colorectal cancer, lung cancer, oesophageal cancer, and glioblastoma [10-14] as well as to differentiate between benign and malignant maxillary sinus masses, cervical lymph nodes, lung nodules, liver masses, adrenal nodules, and renal masses [15-20]. In the current study, there were significant differences in 8 of the 40 texture parameters between CNM-HPV-OPC and $2^{\text {nd }} \mathrm{BC}$. The texture parameters were Histogram Kurtosis, Histogram Entropy log10, Histogram Entropy log2, Histogram Energy, GLRLM GLNU, NGLDM Busyness, GLZLM
LZE, and GLZLM LZHGE. Histogram Kurtosis reflects the shape of the gray-level distribution (peaked or flat) relative to a normal distribution. Histogram Entropy $\log 10$ reflects the randomness of the distribution. Histogram Entropy $\log 2$ reflects the randomness of the distribution. Histogram Energy reflects the uniformity of the distribution. GLRLM GLNU reflects the non-uniformity of the grey-levels or the length of the homogeneous runs. NGLDM Busyness reflects the spatial frequency of changes in intensity. GLZLM LZE reflects the distribution of the short or long homogeneous zones in an image. Finally, GLZLM LZHGE reflects the distribution of the long homogeneous zones with low or high grey-levels. The cutoff values of these parameters may help in the differential diagnosis of these diseases.

There were several limitations to our study. This study was retrospective and limited by the small number of cases at the same institution. Because our study was limited to a single race population, potential genetic components could not be eliminated.

\section{Conclusions}

There were significant differences between CNM-HPV$\mathrm{OPC}$ and $2^{\text {nd }} \mathrm{BC}$ with regard to $\mathrm{CT}$ imaging findings and textural parameters. These results may help in differentiating the 2 conditions. Further study is recommended to confirm their clinical significance.

\section{Conflict of interest}

The authors report no conflict of interest. 


\section{Reference}

1. Ridge JA, Lydiatt WM, Patel SG, et al. Lip and oral cavity. In: Amin MB, Edge S, Greene F, et al. (eds.). AJCC Cancer Staging Manual. $8^{\text {th }}$ ed. New York, NY: Springer; 2017; 79-94.

2. Goyal N, Zacharia TT, Goldenberg D. Differentiation of branchial cleft cysts and malignant cystic adenopathy of pharyngeal origin. Am J Roentgenol 2012; 199: 216-221.

3. Rath TJ, Narayanan S, Hughes MA, et al. Solid lymph nodes as an imaging biomarker for risk stratification in human papillomavirus-related oropharyngeal squamous cell carcinoma. Am J Neuroradiol 2017; 38: 1405-1410.

4. Marur S, D'Souza G, Westra WH, et al. HPV-associated head and neck cancer: A virus-related cancer epidemic. Lancet Oncol 2010; 11: 781-789.

5. Windon MJ, D'Souza G, Rettig EM, et al. Increasing prevalence of human papillomavirus-positive oropharyngeal cancers among older adults. Cancer 2018; 124: 2993-2999.

6. Goldenberg D, Begum S, Westra W, et al. Cystic lymph node metastasis in patients with head and neck cancer: an HPV-associated phenomenon. Head Neck 2008; 30: 898-903.

7. Chan MW, Yu E, Bartlett E, et al. Morphologic and topographic radiologic features of human papillomavirus-related and -unrelated oropharyngeal carcinoma. Head Neck 2017; 39: 1524-1534.

8. Morani AC, Eisbruch A, Carey TE, et al. Intranodal cystic changes: a potential radiologic signature/biomarker to assess the human papillomavirus status of cases with oropharyngeal malignancies. J Comput Assist Tomogr 2013; 37: 343-345.

9. Incoronato $\mathrm{M}$, Aiello $\mathrm{M}$, Infante $\mathrm{T}$, et al. Radiogenomic analysis of oncological data: A technical survey. Int J Mol Sci 2017; 18: 1-28.

10. Kuno H, Qureshi MM, Chapman MN, et al. CT texture analysis potentially predicts local failure in head and neck squamous cell carcinoma treated with chemoradiotherapy. Am J Neuroradiol 2017; 38: 2334-2340.

11. Liu Y, Xu X, Yin L, et al. Relationship between glioblastoma heterogeneity and survival time: An MR imaging texture analysis. Am J Neuroradiol 2017; 38: 1695-1701.
12. Yip C, Landau D, Kozarski R, et al. Primary esophageal cancer: heterogeneity as potential prognostic biomarker in patients treated with definitive chemotherapy and radiation therapy. Radiology 2013; 270 : 122869 .

13. Miles KA, Ganeshan B, Griffiths MR, et al. Colorectal cancer: texture analysis of portal phase hepatic CT images as a potential marker of survival. Radiology 2009; 250: 444-452.

14. Kim H, Park CM, Keam B, et al. The prognostic value of CT radiomic features for patients with pulmonary adenocarcinoma treated with EGFR tyrosine kinase inhibitors. PLoS One 2017; 12: e0187500.

15. Ramkumar S, Ranjbar S, Ning S, et al. Mri-based texture analysis to differentiate sinonasal squamous cell carcinoma from inverted papilloma. Am J Neuroradiol 2017; 38: 1019-1025.

16. Kuno H, Garg N, Qureshi MM, et al. CT texture analysis of cervical lymph nodes on contrast-enhanced [18F] FDG-PET/CT images to differentiate nodal metastases from reactive lymphadenopathy in HIV-positive patients with head and neck squamous cell carcinoma. Am J Neuroradiol 2019; 40: 543-550.

17. Dennie C, Thornhill R, Sethi-Virmani V, et al. Role of quantitative computed tomography texture analysis in the differentiation of primary lung cancer and granulomatous nodules. Quant Imaging Med Surg 2016; 6: 6-15.

18. Stocker D, Marquez HP, Wagner MW, et al. MRI texture analysis for differentiation of malignant and benign hepatocellular tumors in the non-cirrhotic liver. Heliyon 2018; 4: e00987.

19. Ho LM, Samei E, Mazurowski MA, et al. Can Texture analysis be used to distinguish benign from malignant adrenal nodules on unenhanced CT, contrast-enhanced CT, or in-phase and opposed-phase MRI? Am J Roentgenol 2019; 212: 554-561.

20. Deng Y, Soule E, Cui E, et al. Usefulness of CT texture analysis in differentiating benign and malignant renal tumours. Clin Radiol 2020; 75: 108-115. 